Pacific Journal of Mathematics

SINGULAR INTEGRALS IN SEVERAL VARIABLES OVER A 


\section{SINGULAR INTEGRALS IN SEVERAL VARIABLES OVER A LOCAL FIELD}

\section{Keith Phillips and Mitchell Taibleson}

In this paper we construct singular integral transforms of the Calderón-Zygmund type for $K^{d}$ where $K$ is a nondiscrete zero dimensional locally compact field and $d$ is a positive integer. The transforms have the form

$$
L f=\lim _{k \rightarrow \infty} \psi_{k} * f,
$$

where the kernel $\psi_{k}$ vanishes in a neighborhood of 0 ,

$$
\int_{|x|=1} \psi_{k}(x) d x=0
$$

and $\psi_{k}$ satisfies certain smoothness conditions.

The results generalize the results of [6] in several ways: the $p$ adic and $p$-series fields are replaced with $K^{d}$, pointwise convergence is proved, and the hypothesis on the kernels is weakened. Many of the methods also apply in other settings; see, e.g., the second author's forthcoming paper on multipliers [11], and the argument in Lemma 10 [12, p. 201].

We let $Z$ denote the integers, $Z^{+}$the positive integers. The complement of a set $S$ is denoted by $S^{\prime}$, its characteristic function by $\xi_{s}$. In general, our notation for the locally compact field $K$ is as in [9]. The second section of [9] also includes a good summary of that elementary analysis on $K$ which we will need; $K^{d}$ is treated in the first pages of [12]. Let $m$ be the modular function for $K(\lambda(a S))=$ $m(a) \lambda(S), \lambda$ Haar measure for $(K,+))$. We also let $|x|=m(x)$. The sets

$$
\mathfrak{P}^{0}=\{x:|x| \leqq 1\} \text { and } \mathfrak{P}^{1}=\{x:|x|<1\}
$$

are the ring of integers of $K$ and the unique maximal ideal of $\mathfrak{P}^{\circ}$, respectively. Let $o\left(\mathfrak{P}^{0} / \mathfrak{P}^{1}\right)=p^{a}=q(p$ prime $)$ and $\mathfrak{P}^{1}=(\pi)$. For $s \in Z$, $\left|\pi^{s}\right|=q^{-s}$. Every $\alpha \in K \backslash\{0\}$ has a unique representation

$$
\alpha=\pi^{s} \alpha^{*} \quad \text { with } \quad s=s(\alpha) \in Z \text { and }\left|\alpha^{*}\right|=1 \text {. }
$$

The vector space $K^{d}$ is all $d$-tuples of elements of $K$. We use the norm

$$
N(x)=|x|=\sup \left\{\left|x_{i}\right|: 1 \leqq i \leqq d\right\}\left(x=\left(x_{i}\right)\right),
$$

which is easily seen to be non-Archimeadean: $|x+y| \leqq \max [|x|,|y|]$. Any $x \in K^{d} \backslash\{0\}$ can be written uniquely in the form 


$$
x=\pi^{s} x^{*} \quad \text { with } \quad s=s(x) \in Z \text { and }\left|x^{*}\right|=1 \text {. }
$$

we let

$$
\mathfrak{P}^{n}=\left\{x \in K^{d}:|x| \leqq q^{-n}\right\} ; \quad \mathfrak{Q}^{n}=\left\{x:|x|=q^{-n}\right\} .
$$

Each $\mathfrak{P}^{n}$ is a subgroup of $K^{d}$ and $\left\{\mathfrak{P}^{n}\right\}_{n=0}^{\infty}$ is a neighborhood basis at 0 . We use the inner product

$$
\langle x, y\rangle=\sum_{i=1}^{d} x_{i} y_{i} .
$$

There is a character $\chi$ on $(K,+)$ which is identically one on $\mathfrak{S}^{0}$ but is nontrivial on the group $\mathfrak{\beta}^{-1}=\{\alpha:|\alpha| \leqq q\}$. If we let $\chi_{\beta}(\alpha)=\chi(\alpha \beta)$, then the mapping $\beta \rightarrow \chi_{\beta}$ is a topological isomorphism of $(K,+)$ onto its dual $D(K,+)$; we thus identify $(K,+)$ and $D(K,+)$. Letting $\chi_{y}(x)=\chi(\langle x, y\rangle)$ for $x, y \in K^{d}$, it follows that $y \rightarrow \chi_{y}$ is a topological isomorphism of $K^{d}$ onto $D\left(K^{d}\right)$. The anihilator in $D\left(K^{d}\right)$ of $\mathfrak{P}^{n}$ is $A\left(\mathfrak{P}^{n}\right)=$ $\left\{\chi_{y}: \chi_{y}(x)=1\right.$ for all $\left.x \in \mathfrak{P}^{n}\right\}$. Hence,

$$
A\left(\mathfrak{P}^{n}\right)=\left\{\chi_{y}: y \in \mathfrak{P}^{-n}\right\}\left(=\mathfrak{P}^{-n}\right), \quad n \in Z .
$$

Normalization of Haar measure $\lambda$ on $K^{d}$ so that the companion Haar measure on $D\left(K^{d}\right)$ making inversion theorems and Plancherel's theorem valid is again $\lambda$ requires that $\lambda\left(A\left(\mathfrak{P}^{n}\right)\right) \lambda\left(\mathfrak{P}^{n}\right)=1$. (This is an easy calculation. See also (2.3) of [6]). In particular, $\lambda\left(\mathfrak{P}^{0}\right)=1$. The Fourier transform of a function $f$ on $K^{d}$ is denoted by $\hat{f}$; it is initially defined on $\mathfrak{Q}_{1}$ by $\hat{f}(y)=\int_{K^{d}} f(x) \overline{\chi(\langle x, y\rangle)} d x$. The inverse Fourier transform is denoted by $\check{f}$ :

$$
\check{f}(y)=\int_{K^{d}} f(x) \chi(\langle x, y\rangle) d x .
$$

The symbols $\mathfrak{C}, \mathfrak{S}_{0}$, and $\mathfrak{Z}_{r}(1 \leqq r \leqq \infty)$ denote the usual function spaces, defined for $\left(K^{d}, \lambda\right)$ if not otherwise indicated; $\mathfrak{S}_{00}$ is the continuous functions with compact support. In this and in any unexplained notation, we follow [4]. For $1<r<\infty, r^{\prime}$ denotes the numbers such that $1 / r+1 / r^{\prime}=1$. The function space $\mathfrak{S}$ is all $\phi \in \mathfrak{S}_{00}$ for which there is some $n$ such that $\varphi\left(x+\mathfrak{P}^{n}\right)=\varphi(x)$, all $x \in K^{d}$.

Some often used computational principles are worth mentioning at the outset. First, if $f \in \mathfrak{R}_{1}\left(K^{d}, \lambda\right)$, we can write

$$
\int_{K^{d}} f d \lambda=\sum_{j=-\infty}^{\infty} \int_{0 j} f d \lambda \text {. }
$$

Second, since $\lambda$ is the product of $d$ factors of Haar measure for $K$ and since the multiplicative Haar integral for $K$ is $f \rightarrow \int_{K}(f / m) d \lambda$, we have 


$$
\int_{K^{d}} f(\alpha x) d x=\frac{1}{|\alpha|^{d}} \int_{K^{d}} f(x) d x
$$

if $\alpha \in K \backslash\{0\}$ and $f \in \mathbb{R}_{1}\left(K^{d}, \lambda\right)$. Combining these, we have that

$$
\int f(x)|x|^{-d} d x=\sum_{j=-\infty}^{\infty} \int_{\Sigma_{0}^{0}} f\left(\pi^{-j} x\right) d x .
$$

We also often use the fact that

$$
\int_{\mathfrak{Q}^{j}} \chi(\langle x, y\rangle) d y=0 \quad \text { when } \quad|x|>q^{-j}
$$

2. Lebesgue set; maximal functions. The proof of pointwise convergence in $\S 3$ depends strongly on the Lebesgue set of a function and on maximal functions. Both of these ideas can be developed in in considerable generality, and we will do this in a section which is independent of the rest of the paper, $\S 4$. However the facts for $K^{d}$ are considerably easier and we present them here. The set of $x$ for which (2.1.i) holds will be called, as in the classical case, the Lebesgue set for $f$.

TheOREM 2.1. Let $f \in \mathfrak{R}_{r, \mathrm{loc}}\left(K^{d}\right), r>1$. For almost all $x$, we have (i ) $\lim _{n \rightarrow \infty} \frac{1}{\lambda\left(\mathfrak{P}^{n}\right)} \int_{\mathfrak{P}^{n}}|f(x \pm y)-f(x)|^{r} d y=0$;

(ii) $\lim _{n \rightarrow \infty} \frac{1}{\lambda\left(\mathfrak{P}^{n}\right)} \int_{\mathfrak{P}^{n}}|f(x+y)+f(x-y)-2 f(x)|^{r} d y=0$.

Proof. By differention of indefinite integrals (see (2.9) of [3]), we have

$$
\lim _{n \rightarrow \infty} \frac{1}{\lambda\left(\mathfrak{P}^{n}\right)} \int_{\mathfrak{P}^{n}}|f(x+y)-\alpha|^{r} d y=|f(x)-\alpha|^{r} \text { a.e . }
$$

for each complex number $\alpha$ with rational coordinates. The proof of (i) is completed as in the classical case (see, e.g., [13], p. 65); (ii) follows from (i).

REMark 2.2. The result of Edwards and Hewitt used above applies to a general class of locally compact groups, and the proof is fairly involved. The situation is much simpler for $K^{d}$ (or for any first countable zero dimensional group), as described in the next few lines.

The equality we want is

$$
\text { (*) } \quad \lim _{n \rightarrow \infty} \frac{1}{\lambda\left(\mathfrak{B}^{n}\right)} \int_{x+\mathfrak{F}^{n}} f(y) d y=f(x) \quad \text { a.e . }
$$

for $f \in \mathfrak{Q}_{1,1 \mathrm{loc}}\left(K^{d}, \lambda\right)$. We will prove $\left(^{*}\right)$ using (2.3), below. 
Let $f \in \mathbb{R}_{1, \mathrm{loc}}^{+}\left(K^{d}, \lambda\right)$. For maximal functions, we use the notation

$$
M_{n} f(x)=\frac{1}{\lambda\left(\mathfrak{P}^{n}\right)} \int_{x+\mathfrak{P}^{n}} f d \lambda, n \in Z ; M f(x)=\sup _{n \in Z} M_{n} f(x) .
$$

For a function $g \geqq 0$ and $t \geqq 0$, let $E_{t}[g]=\{x: g(x)>t\}$.

THEOREM 2.3. If $f \in \mathbb{R}_{r}^{+}\left(K^{d}\right)(1 \leqq r<\infty)$ and $t>0$, then

$$
\lambda\left(E_{t}[M f]\right) \leqq \frac{1}{t} \int \xi_{E_{t}[M f]} f d \lambda .
$$

Proof. If $x \in E_{t}[M f]$, there is a $\mathfrak{P}^{n_{x}}$ such that $\int_{x+\mathfrak{P}^{n} x} f d \lambda>t \lambda\left(\mathfrak{P}^{n^{n}}\right)$. Since $y+\mathfrak{B}^{n_{x}}=x+\mathfrak{P}^{n_{x}}$ if $y \in x+\mathfrak{B}^{n_{x}}$, we have $x+\mathfrak{B}^{n_{x}} \subset E_{t}[M f]$. A pair of cosets are either disjoint or one is contained in the other. It follows that there is a pairwise disjoint family $\left\{x_{n}+\mathfrak{P}^{k_{n}}\right\}_{n=1}^{\infty}$ such that $E_{t}[M f]=\bigcup_{n=1}^{\infty}\left(x_{n}+\mathfrak{S}^{k}{ }^{k}\right)$ and

$$
\int_{x_{n}+\Re^{k} n} f d \lambda>t \lambda\left(\mathfrak{P}^{k n}\right)
$$

The equality (i) follows.

We now prove $(*)$. We may assume that $f \in \mathfrak{R}_{1}$. Choose $t>0$, and $g$ a continuous function that $\int|g-f|<t^{2} / 4$. Then

$$
\begin{aligned}
H(x)= & \lim \sup _{n \rightarrow \infty}\left|\frac{1}{\lambda\left(\mathfrak{P}^{n}\right)} \int_{x+\mathfrak{F}^{n}}(f(y)-f(x)) d y\right| \\
\leqq & \lim \sup _{n \rightarrow \infty}\left|\frac{1}{\lambda\left(\mathfrak{P}^{n}\right)} \int_{x+\mathfrak{F}^{n}}(g(y)-g(x)) d y\right| \\
& +\lim \sup _{n \rightarrow \infty}\left|\frac{1}{\lambda\left(\mathfrak{P}^{3}\right)} \int_{x+\mathfrak{F}^{n}}\right| f(y)-g(y)|d y+| f(x)-g(x) \mid \\
\leqq & M(|f-g|)(x)+|f-g|(x) .
\end{aligned}
$$

From (2.3) it follows that

$$
\begin{aligned}
\left.\lambda\left(E_{t} \mid H\right]\right) & \left.\leqq \lambda\left(E_{t / 2}[M|f-g|]\right)+\lambda\left(E_{t / 2}|| f-g \mid\right]\right) \\
& \leqq \frac{2}{t} \int|f-g|+\frac{2}{t} \int|f-g|=\frac{4}{t} \cdot \frac{t^{2}}{4}=t .
\end{aligned}
$$

It follows that $H(x)=0$ a.e. and the equality $(*)$ follows.

2.4. Maximal function inequalities.

(i) $\|M f\|_{r} \leqq \frac{r}{r-1}\|f\|_{r}$ if $f \in \mathbb{Q}_{r}^{+}(1<r<\infty)$.

(ii) $\int_{E}[M f] d \lambda \leqq \frac{1}{k} \lambda(E)+\frac{1}{1-k} \int_{K^{d}} f\left[\log ^{+} f\right] d \lambda$, for any $f \in L_{1}^{+}$, 
any $k \in] 0,1[$, any $\lambda$-measurable $E$.

(iii) $\int[M f]^{r} d \lambda \leqq \frac{\lambda(E)^{1-r}}{1-r}\left(\int_{K^{d}} f d \lambda\right)^{r}$ for $\left.r \in\right] 0,1\left[\right.$, any $f \in \mathfrak{R}_{1}^{+}$, and any $\lambda$-measurable $E$.

These inequalities follow from (2.3.i); see, e.g., (2.2), (3.1), (3.2), and (3.4) of [7].

For equations dealing with pointwise convergence, we need some more technical results about maximal functions, which follow. The reader might prefer to read on to part IV of the proof of (3.1), where the results are first needed.

Let $\zeta=\xi_{\mathfrak{R}^{0}}$, for convenience. The average $M_{n} f$ for $f \in L_{r}^{+}(1 \leqq$ $r<\infty)$ can be written

$$
M_{n} f(x)=\frac{1}{\lambda\left(\mathfrak{P}^{n}\right)} \int_{K^{d}} \zeta\left(\pi^{-n}(y-x)\right) f(y) d y .
$$

Replacing $\zeta$ with a measurable function $\eta$, we define (formally)

$$
M_{n}(\eta, f)(x)=\frac{1}{\lambda\left(\mathfrak{P}^{n}\right)} \int \eta\left(\pi^{-n}(y-x)\right) f(y) d y ; M(\eta, f)=\sup _{n \in Z}\left|M_{n}(\eta, f)\right| .
$$

Technical Lemma 2.5. For a measurable function $\eta$ on $K^{d}$, let $\varphi(x)=\varphi(|x|)=\sup \{|\eta(z)|:|z|=|x|\}$. We have

( i ) $M(\eta, f) \leqq\|\varphi\|_{1} M(|f|)$ for $f \in \mathbb{R}_{r}(1 \leqq r<\infty)$. If $\eta \in \mathfrak{R}_{1}$ and satisfies

(ii) $\sum_{j=-\infty}^{\infty}\left[\int_{\mathbb{Q}^{j}}|\eta(x)|^{r^{\prime}} d x\right]^{1 / r^{\prime}} \lambda\left(\mathfrak{P}^{j}\right)^{1 / r}<\infty$ for some $\left.r \in\right] 0, \infty[$, then for $f \in \mathfrak{R}_{r}$, we have

(iii) $\lim _{n \rightarrow \infty} M_{n}(\eta, f)(x)=f(x) \int \eta d \lambda$ a.e.;

(iv) $|M(\eta, f)(x)| \leqq b\left[M\left(|f|^{r}\right)(x)\right]^{1 / r}$ a.e., where $b$ is a constant. If $\phi \in \mathfrak{R}_{1}$, then (ii) holds for all $r>1$, and so (iii) and (iv) hold in this case also.

Proof. Suppose first that $\eta \in \mathbb{\Omega}_{1}$ and satisfies (ii). Write

$$
M_{n}(\eta, f)(x)=\frac{1}{\lambda\left(\mathfrak{B}^{n}\right)} \int \eta\left(\pi^{-n}(y-x)\right)[f(y)-f(x)] d y+f(x) \int \eta d y,
$$

to see that

$$
\left|M_{n}(\eta, f)(x)-f(x) \int \eta d \lambda\right| \leqq \frac{1}{\lambda\left(\mathfrak{B}^{n}\right)} \int \eta\left(\pi^{-n} y\right)|f(x+y)-f(x)| d y
$$


(1)

$$
\begin{aligned}
\leqq & \sum_{j=-\infty}^{\infty}\left[\int_{\mathfrak{Q}^{j-n}}|\eta|^{r^{\prime}} d \lambda\right]^{1 / r^{\prime}} \frac{\left|\pi^{n}\right| d / r^{\prime} \lambda\left(\mathfrak{P}^{j}\right)^{1 / r}}{\lambda\left(\mathfrak{P}^{n}\right)} \\
& {\left[\frac{1}{\lambda\left(\mathfrak{P}^{j}\right)} \int_{\mathfrak{Q}^{j}}|f(x+y)-f(x)|^{r} d y\right]^{1 / r} } \\
\sum_{j=-\infty}^{\infty}\left[\int_{\mathfrak{Q}^{j-n}}|\eta|^{r^{\prime}} d \lambda\right]^{1 / r^{\prime}} \lambda\left(P^{j-n}\right)^{1 / r} & {\left[\frac{1}{\lambda\left(\mathfrak{P}^{j}\right)} \int_{\mathfrak{Q}^{j}}|f(x+y)-f(x)|^{r} d y\right]^{1 / r} . }
\end{aligned}
$$

The last bracketed term-[ $]^{1 / r}$-is bounded by $\|f\|_{r}+|f(x)|$ if $j<0$; and, for $x$ in the Lebesgue set for $f$, it is bounded for $j \geqq 0$. Suppose $x$ is in the Lebesgue set, let $c$ bound the term for all $j \in Z$, and let $S_{J}$ be its supremum over $j \geqq J, J \in Z$. The last term in (1) is thus dominated by

$$
S_{J} \sum_{j=J-n}^{\infty}\left[\int_{\mathbb{Q}^{j}}|\eta|^{r^{\prime}} d \lambda\right]^{1 / r^{\prime}} \lambda\left(\mathfrak{P}^{j}\right)^{1 / r}+c \sum_{j=-\infty}^{J-n-1}\left[\int_{Q^{j}}|\eta|^{r^{\prime}}\right]^{1 / r^{\prime}} \lambda\left(\mathfrak{P}^{j}\right)^{1 / r} .
$$

The second term goes to 0 as $n$ goes to $\infty$, because of (ii); and, the bound becomes $S_{J} \sum_{j=-\infty}^{\infty}\left[\int_{Q^{j}}|\eta|^{r^{\prime}} d \lambda\right]^{1 / r^{\prime}} \lambda\left(\Re^{j}\right)^{1 / r}$. As $J$ goes to $\infty$, this expression goes to 0 ; and (iii) is established.

To prove (iv), use the same kind of estimates to write

$$
\begin{aligned}
& \left|M_{n}(\eta, f)(x)\right| \\
& \quad \leqq \sum_{j=-\infty}^{\infty}\left[\int_{\mathbb{O}^{j-n}}|\eta|^{r^{\prime}}\right]^{1 / r^{\prime}} \lambda\left(P^{j-n}\right)^{1 / r}\left[\frac{1}{\lambda\left(\mathfrak{P}^{j}\right)} \int_{\mathbb{Q}^{j}}|f(x+y)|^{r} d y\right]^{1 / r} \\
& \quad \leqq b\left[M\left(|f|^{r}\right)(x)\right]^{1 / r} .
\end{aligned}
$$

To prove (i), estimate as follows:

$$
\begin{aligned}
\left|M_{n}(\eta, f)(x)\right| & \leqq \sum_{j=-\infty}^{\infty} \varphi\left(\pi^{-n+j}\right) \lambda\left(P^{j-n}\right) \frac{1}{\lambda\left(\mathfrak{P}^{j}\right)} \int_{\mathfrak{Q}^{j}}|f(x+y)| d y \\
& \leqq\left[\int_{K^{d}} \varphi d \lambda\right] M(|f|)(x) .
\end{aligned}
$$

Finally, " $\varphi \in \mathfrak{Q}_{1}$ " can be stated " $\sum_{j=-\infty}^{\infty}\left\{\sup _{|x|=q^{-}}|\eta(x)|\right\} \lambda\left(\mathfrak{\Omega}^{j}\right)<\infty "$. If this holds, then

$$
\begin{aligned}
\sum_{j=-\infty}^{\infty} & \left\{\int_{\Omega^{j}}|\eta|^{r^{\prime}} d \lambda\right\}^{1 / r^{\prime}} \lambda\left(\Omega^{j}\right)^{1 / r} \\
& \leqq \sum_{j=-\infty}^{\infty}\left\{\sup _{|x|=q^{-j}}|\eta(x)|^{r^{\prime}} \lambda\left(\Omega^{j}\right)\right\}^{1 / r^{\prime}} \lambda\left(\Omega^{j}\right)^{1 / r}<\infty,
\end{aligned}
$$

so that (ii) holds. Obviously $\varphi \in \mathfrak{R}_{1} \Rightarrow \eta \in \mathfrak{R}_{1}$, so (iii) and (iv) hold.

REMARK. The preceding lemma is local a field variant of Lemmas 1,2 , and 3 of Chapter II in [2]. 
3. Singular integrals. Our main theorem is stated and proved below. In (3.2) and (3.3) we give variants. In (3.4) there is a discussion of various aspects of the hypothesis and an indication of a simpler proof for smoother $\omega$.

THEoRem 3.1. Suppose $\omega \in \mathfrak{R}_{\infty}\left(K^{d}\right), \omega\left(\pi^{s} x\right)=\omega(x)$ for $s \in Z$, and $\int_{\mathfrak{Q}^{0}} \omega(x) d x=0 . \quad$ Define

$$
\psi_{k}(x)=\frac{\omega(x)}{|x|^{d}} \xi_{\left(\mathfrak{F}^{k+1}\right)^{\prime}}(x), k \in Z .
$$

If the condition

(i ) $\quad \sup _{y \in \mathfrak{Q}^{0}} \sum_{j=1}^{\infty} \int_{\mathfrak{Q}^{0}}\left|\omega\left(x+\pi^{j} y\right)-\omega(x)\right| d x<\infty$

is satisfied, then for each $r \in] 1, \infty\left[\right.$ there is a constant $A_{r}$ such that

$$
\left\|f * \psi_{k}\right\|_{r} \leqq A_{r}\|f\|_{r}
$$

holds and $L f=\lim _{k \rightarrow \infty} f * \psi_{k}$ exists in $\mathfrak{R}_{r}$-norm for $f \in \mathfrak{R}_{r}$. If $f \in \mathfrak{R}_{1}$, then there is a constant $A_{1}$ such that $\lambda\left(E_{t}\left[f * \psi_{k}\right]\right) \leqq A_{1} t^{-1}\|f\|_{1}$ (the convolution operators are uniformly weak type $(1,1)), L f=\lim _{k \rightarrow \infty} f * \psi_{k}$ exists in measure, and $\lambda\left(E_{t}[L f]\right) \leqq A_{1} t^{-1}\|f\|_{1}$.

If in addition to (i), the condition

$$
\sum_{j=1}^{\infty} \sup _{x \in \mathfrak{\Sigma}^{0}}\left\{\int_{\mathfrak{P}^{0}}\left|\omega\left(x+\pi^{j} y\right)-\omega(x)\right| d y\right\}<\infty
$$

is satisfied, then for each $r \in\left[1, \infty\right.$ [the sequence $\left(\psi_{k} * f\right)_{k=1}^{\infty}$ converges pointwise a.e. for $f \in \mathfrak{Q}_{r}$ and for $\left.r \in\right] 1, \infty$ [there is a constant $B_{r}$ such that $L^{*} f=\sup _{k \in Z^{+}}\left|\psi_{k} * f\right|$ satisfies

$$
\left\|L^{*} f\right\|_{r} \leqq B_{r}\|f\|_{r}, \text { all } f \in L_{r} .
$$

The operator $L^{*}$ is weak type $(1,1)$.

Proof. I. $\mathfrak{R}_{2}$-convergence. The proof of $\mathfrak{R}_{2}$-convergence is based on inversion of $\Omega_{2}$-Fourier transforms, and this argument depends on uniform boundedness of $\left(\hat{\psi}_{k}\right)_{k=1}^{\infty}$. (The functions $\psi_{k}$ are in $\mathbb{L}_{s}$ for $s \in] 1, \infty]$; see (2.1) of [6]). For $n<0$ and $k \geqq 0$, let

$$
{ }_{n} \psi_{k}=\psi_{k} \xi_{\mathfrak{F}^{n}}\left(=\frac{\omega}{|\cdot|^{d}} \xi_{\mathfrak{F}^{n} \cap\left(\mathfrak{F}^{k+1}\right)^{\prime}}\right) .
$$

Each ${ }_{n} \psi_{k}$ is in $\mathfrak{R}_{1}$ (because $\left.\omega \in \mathfrak{R}_{\infty}\right)$. Since $A\left(\mathfrak{P}^{n}\right)=\mathfrak{P}^{-n},(2.3)$ of [6] implies that

$$
\left\{\psi_{0}(x-v)\right\}^{\wedge}(y)=\lim _{n \rightarrow-\infty} \int_{\mathfrak{F}^{n}} \psi_{0}(x) \overline{\chi(\langle x, y\rangle+\langle v, y\rangle)} d x
$$

a.e. in $y$ for each $v \in K^{d}$. 
(The notation $\left\{\psi_{0}(x-v)\right\}$ means the function with value $\psi_{0}(x-v)$ at $x)$. Thus,

$$
\left.\left\{\psi_{0}(x-v)-\psi_{0}(x)\right\}^{\wedge}(y)=\overline{[\chi(\langle v, y\rangle)}-1\right] \hat{\psi}_{0}(y) \text { a.e. in } y \text {. }
$$

For $v \in \mathfrak{B}^{0}$, the functions $\left\{\psi_{0}(x-v)-\psi_{0}(x)\right\}$ are in $\mathfrak{\Omega}_{1}$ and have uniformly bounded $\&_{1}$-norms (see (i)). Thus $\left\{\psi_{0}(x-v)-\psi_{0}(x)\right\}^{\wedge} \in \mathfrak{S}_{0}$ are uniformly bounded when $|v| \leqq 1$. If $|y|>1$, the function $v \rightarrow|\overline{\chi(\langle v, y\rangle)}-1|$ attains a positive maximum $M$, say at $v_{y}$, on $\mathfrak{P}^{0}$. The same value is attained for $\left(\pi^{-s} y, \pi^{s} v_{y}\right)(s \geqq 0)$. Thus, for each $y \in\left(\mathfrak{P}^{0}\right)^{\prime}$ for which (2) holds there is a $v_{y} \in \mathfrak{P}^{0}$ such that

$$
\left|\hat{\psi}_{0}(y)\right|=M^{-1}\left|\left\{\psi_{0}\left(x-v_{y}\right)-\psi_{0}(x)\right\}^{\wedge}(y)\right| .
$$

The right side of $\left.\| \psi_{0}\left(x-v_{y}\right)-\psi_{0}(z)\right\}^{\wedge}\left\|_{\infty} \leqq\right\|\left\{\psi_{0}\left(x-v_{y}\right)-\psi_{0}(x)\right\} \|_{1}$ is bounded (by (i)), so there is a constant $B$ such that

$$
\left|\hat{\psi}_{0}(y)\right| \leqq B \quad \text { a.e. in }\left(\mathfrak{F}^{0}\right)^{\prime} \text {. }
$$

(This boundedness argument is a modification of one appearing in Hörmander [5]). We have

(4) $\quad \hat{\psi}_{0}(y)-\hat{\psi}_{0}\left(\pi^{-s} y\right)=\sum_{j=1}^{s} \int_{\mathfrak{Q}^{0}} \omega(x) \overline{\chi\left(\pi_{j}\langle x, y\rangle\right)} d x \quad$ a.e. in $y, s \in Z^{+}$.

If $y \in \mathfrak{\Omega}^{s-1}$, (4) shows that $\hat{\psi}_{0}(y)=\hat{\psi}_{0}\left(\pi^{-s} y\right)$; and, $\pi^{-s} y \in \mathfrak{Z}^{-1}$. Thus, by (3), $\hat{\psi}_{0} \in \mathbb{R}_{\infty}$.

The equality $\hat{\psi}_{k}(y)=\hat{\psi}_{0}\left(\pi^{k} y\right)$ shows that $\left\{\left\|\hat{\psi}_{k}\right\|_{\infty}\right\}_{k=0}^{\infty}$ is uniformly bounded and that

$$
\hat{\psi}_{k}(y)=\sum_{j=-\infty}^{\min (-1, k+s(y))} \int_{\mathfrak{Q}^{0}} \omega(x) \overline{\chi\left(\pi^{j}\left\langle x, y^{*}\right\rangle\right)} d x \text { a.e. }
$$

Thus,

$$
\varphi(y)=\lim _{k \rightarrow \infty} \hat{\psi}_{k}(y)=\sum_{j=-\infty}^{-1} \int_{\Omega_{0}^{0}} \omega(x) \overline{\chi\left(\pi^{j}\left\langle x, y^{*}\right\rangle\right.} d x
$$

exists a.e. Finally, the equality $\hat{\psi}_{k}(y)-{ }_{n} \hat{\psi}_{k}(y)=\hat{\psi}_{0}\left(\pi^{n-1} y\right)(n<0, k \geqq 0)$ shows that $\left\{\left\|\hat{\psi}_{k}-{ }_{n} \hat{\psi}_{k}\right\|_{\infty}: n<0, k \geqq 0\right\}$ is bounded by any constant bounding $\left\{\left\|\hat{\psi}_{k}\right\|_{\infty}: k>0\right\}$.

The $\mathfrak{R}_{2}$-convergence argument stemming from the above bounds is well known (see [2] or [6]) and goes as follows. If $f \in \mathbb{R}_{2}$, then ${ }_{n} \psi_{k} \hat{f}$ converges $\left(\Omega_{2}\right)$ to $\hat{\psi}_{k} \hat{f}$, inversion gives $\left(\hat{\psi}_{k} \hat{f}\right)^{\vee}=\psi_{k} * f$ a.e., $\hat{\psi}_{k} \hat{f}$ converges $\left(\Omega_{2}\right)$ to $\varphi \hat{f}$, and inversion gives convergence of $\psi_{k} * f$. The bound $\left\|\psi_{k} * f\right\|_{2} \leqq\|\varphi\|_{\infty}\|\hat{f}\|_{2}$ holds; put $A_{2}=\|\varphi\|_{\infty}$ to obtain (ii).

II. Measure estimates; weak type $(1,1)$. Suppose $1 \leqq r \leqq$ $2, f \in \mathfrak{R}_{r}^{+}, k \in Z^{+}$, and $t>0$. Let $E_{t}=E_{t}\left[\psi_{k} * f\right]$. The covering 
lemma (3.12) of [6] states that there is a mapping $(m, n) \rightarrow x_{m n}$ of a subset $P_{t}$ of $Z^{+} \times Z$ into $K^{d}$ such that $\left\{x_{m n}+\mathfrak{P}^{n}:(m, n) \in P_{t}\right\}$ is pairwise disjoint and the following relations hold:

$$
\begin{aligned}
& \text { (a) } t \leqq \frac{1}{\lambda\left(\mathfrak{P}^{n}\right)} \int_{x_{m n}+\mathfrak{P}^{n}} f d \lambda \leqq t q^{d} ; \\
& \text { (b) if } D_{t}=\bigcup\left\{x_{m n}+\mathfrak{P}^{n}:(m, n) \in P_{t}\right\} \text {, then } \lambda\left(D_{t}\right)<\infty(t>0) \\
& \text { and } \lim _{t \rightarrow \infty} \lambda\left(D_{t}\right)=0, \\
& \text { (c) } f(x) \leqq t \text { a.e. in } D_{t}^{\prime} . \\
& \text { (d) } t \lambda\left(D_{t}\right) \leqq \int_{D_{t}} f d \lambda \leqq q^{d} t \lambda\left(D_{t}\right) .
\end{aligned}
$$

As in [6] and [2], we will prove that there are constants $c_{1}$ and $c_{2}$ depending only on $K^{d}$ and $\omega$ such that

$$
\lambda\left(E_{t}\right) \leqq \frac{c_{1}}{t^{2}} \int_{K^{d}}[f]_{t}^{2} d \lambda+c_{2} \lambda\left(D_{t}\right)
$$

where, $[f]_{t}(x)=f(x)$ if $f(x) \leqq t$ and $[f]_{t}(x)=t$ if $f(x)>t$. This will also prove that $\psi_{k} * f$ is uniformly weak type $(1,1)$. We split $f$ by writing $f(x)=h(x)+g(x)$, where $h(x)=f(x)$ if $x \in D_{t}^{\prime}$ and

$$
h(x)=\frac{1}{\lambda\left(\mathfrak{P}^{n}\right)} \int_{x_{m n}+\mathfrak{P}^{n}} f d \lambda \quad \text { if } \quad x \in x_{m n}+\mathfrak{P}^{n} .
$$

To estimate $\lambda\left(E_{t}\right)$, consider

$$
E_{t}^{1}=\left\{x:\left|\psi_{k} * h(x)\right|>\frac{t}{2}\right\} \text { and } E_{t}^{2}=\left\{x:\left|\psi_{k} * g(x)\right|>\frac{t}{2}\right\} .
$$

The function $h$ is bounded by (a), is in $\mathfrak{R}_{r}^{+}$by (b), is in $\mathfrak{Q}_{2}\left(\|h\|_{2}^{2}<\right.$ $\|h\|_{\infty}^{2-r}\|h\|_{r}^{r}$ ), and satisfies (part I)

$$
\lambda\left(E_{t}^{1}\right) \leqq \frac{4 A_{2}^{2}}{t^{2}} \int_{K^{d}} h^{2} d \lambda .
$$

Since $\int_{K^{d}} h^{2} d \lambda \leqq q^{2 d} t^{2} \lambda\left(D_{t}\right)+\int_{D_{t}^{\prime}}[f]_{t}^{2} d \lambda$ (c above), we have

( 7$) \quad \lambda\left(E_{t}^{1}\right) \leqq \frac{c_{1}}{t^{2}} \int_{K^{d}}[f]_{t}^{2} d \lambda+b \lambda\left(D_{t}\right) \quad\left[c_{1}\right.$ and $b$ constants $]$.

Since $g=0$ on $D_{t}^{\prime}$, we have

$$
\psi_{k} * g(x)=\sum_{P_{t}} \int \xi_{x_{m n}+\Re^{n}}(y) g(y) \psi_{k}(x-y) d y, \quad x \in K^{d} .
$$

(The equality $\int \sum_{P_{t}}=\sum_{P_{t}} \int$ used here is valid by Lebesgue's dominated 
convergence theorem: in $\xi_{\cup_{F^{x} m}+\Re^{n}}(y) g(y) \psi_{k}(x-y)$, let the finite set $F$ expand to $P_{t}$.) Consider the terms of the series for $x \in D_{t}^{\prime}$. If $y \in x_{m n}+$ $\mathfrak{P}^{n}$, then:

$(\alpha) \quad\left(x_{m n}+\mathfrak{P}^{n}\right) \cap\left(x+\mathfrak{P}^{k+1}\right)=\varnothing \Rightarrow|x-y|=\left|x-x_{m n}\right|>q^{-(k+1)} ;$

( $\beta) \quad\left(x_{m n}+\mathfrak{P}^{n}\right) \cap\left(x+\mathfrak{P}^{k+1}\right) \neq \varnothing \Rightarrow x-y \in \mathfrak{P}^{k+1}$.

Thus, using $\int_{x_{m n}+\mathfrak{F}^{n}} g d \lambda=0$, we have

$$
\psi_{k} * g(x)=\sum_{S(x, k)} \int_{x_{m n}+\mathfrak{F}^{n}} g(y)\left[\frac{\omega(x-y)-\omega\left(x-x_{m n}\right)}{\left|x-x_{m n}\right|^{d}}\right] d y,
$$

where $S(x, k)=\left\{(m, n):\left(x_{m n}+\mathfrak{P}^{n}\right) \cap\left(x+\mathfrak{P}^{k+1}\right)=\varnothing\right\}$. Integration over $D_{t}^{\prime}$ and Fubini's theorem give

$$
\begin{aligned}
& \int_{D_{t}^{\prime}}\left|\psi_{k} * g(x)\right| d x \\
& \leqq \sum_{P_{t}} \int_{x_{m n}+\Re^{n}}|g(x)| \int_{K^{d}} \xi_{\left(x_{m n}+\mathfrak{F}^{n}\right)}(x) \frac{\left|\omega(x-y)-\omega\left(x-x_{m n}\right)\right|}{\left|x-x_{m n}\right|^{d}} d x d y .
\end{aligned}
$$

Translating by $x_{m n}$ in the inner integral gives

$$
\sum_{j=s(x)}^{\infty} \int_{m n-y)-n+1}\left|\omega\left(x+\pi^{j}\left(x_{m n}-y\right)^{*}\right)-\omega(x)\right| d x
$$

for that integral. Since $s\left(x_{m n}-y\right) \geqq n$, the hypothesis (i) gives a bound, say $M$, for this series. Thus,

$$
\int_{D_{t}^{\prime}}\left|\psi_{k} * g(x)\right| d x \leqq M \int_{D_{t}}|g| d \lambda .
$$

The bound

$$
\int_{D_{t}}|g| d \lambda \leqq 2 \int_{D_{t}} f d \lambda \leqq 2 q^{d} t \lambda\left(D_{t}\right)
$$

now implies that $\lambda\left(D_{t}^{\prime} \cap E_{t}^{2}\right)<4 M q^{d} \lambda\left(D_{t}\right)$. Thus, $\lambda\left(E_{t}^{2}\right) \leqq a \lambda\left(D_{t}\right)$, a independent of $t, k$, and $f$. This estimate and (7) give (6).

If $f \in \mathfrak{R}_{1}^{+}$, then the first term on the right in (6) is less than $c_{1} t^{-1}\|f\|_{1}$ and the second term is less than $c_{2} t^{-1}\|f\|_{1}$, by (5.d). Thus the operators $f \rightarrow \psi_{k} * f$ are uniformly weak type (1.1). (Our proof is for $f \geqq 0$; for arbitrary $f$, uniform weak type $(1,1)$ follows by writing real $f$ as $\max [f, 0]-(-\min [f, 0])$ and complex $f$ as $f_{1}+i f_{2}$.)

The equality (8) can also be used to prove that $\lim _{k \rightarrow \infty} \psi_{k} * g$ exists in $D_{t}^{\prime}$. Since the series on the right in (8) converges absolutely when $S(x, k)$ is replaced by $P_{t}$ (as we proved above), the relations $S(x, k) \subset$ $S(x, k+1)$ and $\bigcup_{k=1}^{\infty} S(x, k)=P_{t}$ shows that we actually have

$$
\lim _{k \rightarrow \infty} \psi_{k} * g(x)=\sum_{P_{t}} s_{m n}(x)
$$


summands as in (8), for all $x \in D_{t}^{\prime}$. We will use this fact later.

III. $\mathfrak{Q}_{r}$-convergence, $1<r<\infty$. The fact that the operators $f \rightarrow$ $\psi_{k} * f$ are uniformly weak type $(1,1)$ and uniformly weak (in fact strong) type $(2,2)$ implies (by the Marcinkiewicz interpolation theorem; [13], vol. II, p. 112) that they are uniformly strong type $(r, r)$ for every $r \in] 1,2]$; i.e., there are constants $A_{r}$ such that (ii) holds for $f \in \mathfrak{L}_{r}$. (The existence of $A_{r}$ for $\left.\left.r \in\right] 1,2\right]$ can also be proved directly from (6), using a function $f^{*}$ on]0, $\infty$ [that is equimeasurable with $f \in \mathbb{R}_{r}^{+}$and the function $\beta_{f}(s)=s^{-1} \int_{0}^{s} f^{\sharp}(u) d u$. For this method, see [6].) For $r>2$, the bound (ii) is obtained by a duality argument, which we now outline. Suppose $f \in \mathfrak{R}_{r}, r>2$, and $k \in Z^{+}$. Define a functional $T_{f}$ on $\mathfrak{E}_{00}$ by $T_{f} g=\int_{K^{d}} g(x)\left(\psi_{k} * f\right)(x) d x$, and use Fubini's theorem to prove that $T_{f} g=$ $\int_{K^{d}} f(-y)\left(\psi_{k} * g^{\prime}\right)(y) d y\left(g^{\prime}(x)=g(-x)\right)$. This gives $\left|T_{f}(g)\right| \leqq A_{r^{\prime}}\|f\|_{r}\|g\|_{r^{\prime}}$, so that $T_{f}$ has a unique norm preserving extension to $\mathfrak{Q}_{r^{\prime}}$. By duality, the extension is given by an $\mathfrak{Q}_{r}$ function, which has to be $\psi_{k} * f$. The norm of the extension is $\left\|\psi_{k} * f\right\|_{r}$. Hence, $\left\|\psi_{k} * f\right\| \leqq A_{r^{\prime}}\|f\|_{r}$.

To prove $\mathbb{Q}_{r}$ convergence of $\psi_{k} * f$ for all $f \in \mathfrak{S}$, it suffices to prove it for all functions $\xi_{z+\mathfrak{S}^{m}}, z \in K^{d}, m \in Z$. Dominated convergence gives the equality

$$
\begin{aligned}
\lim _{n \rightarrow-\infty} \int \xi_{\mathfrak{F}^{n}}(y) \xi_{z+\mathfrak{F}^{m}}(y) \psi_{k}(x-y) d y & =\int_{z+\mathfrak{F}^{m}} \psi_{k}(x-y) d y \\
& =\int_{(x+z)+\mathfrak{F}^{m}} \psi_{k}(-y) d y
\end{aligned}
$$

for all $x$ and $k$. If $x+z \in \mathfrak{P}^{m}$, (9) equals zero for all $k$. If $x+z \notin \mathfrak{P}^{m}$, then $(x+z)+\mathfrak{P}^{m} \subset\left(\mathfrak{P}^{m}\right)^{\prime}$. Since $\psi_{k}(-y)=\psi_{m-1}(-y)$ when $-y \in\left(\mathfrak{P}^{m}\right)^{\prime}$ and $k \geqq m-1$, we see that $\psi_{k} * \xi_{z+\mathfrak{F}^{m}}(x)=\psi_{m-1} * \xi_{z+\mathfrak{F}^{m}}(x)$ if $k \geqq m$. Convergence in $\mathfrak{L}_{r}$ for $\psi_{k} * \xi_{z+\mathfrak{\beta}^{m}}$ follows at once, and $\mathfrak{Q}_{r}$-convergence for all $\mathfrak{Q}_{r}$ functions $(1<r<\infty)$ is now immediate since $\mathfrak{S}$ is dense in $\mathfrak{Q}_{r}$. Note that we have proved the additional result: if $\sigma \in \mathbb{S}$ is constant on cosets of $\mathfrak{B}^{n}$, then $L \sigma=\psi_{k} * \sigma$ for $k \geqq m-1$. Using this fact and the weak type $(1,1)$ estimate, we get a proof that $\psi_{k} * f$ converges in measure to a function $L f$, if $f \in \mathfrak{R}_{1}$; and, $L f$ satisfies the weak type $(1,1)$ estimate. (We will also use the italized assertion in the proof of pointwise convergence.) We have now established all the assertions of the first paragraph of the theorem.

IV. Pointwise convergence and maximal singular integral. Suppose $f \in \mathfrak{R}_{r}(1<r<\infty)$. First, we summarize some limiting relations:
(a) $\lim _{n \rightarrow \infty} M_{n} L f(x)=L f(x)$ a.e. and
$\lim _{n \rightarrow \infty} M_{n}\left(\psi_{k} * f\right)(x)=\psi_{k} * f(x) \quad$ a.e.
(b) $\lim _{n \rightarrow \infty} M_{n}\left(\psi_{k} * f\right)(x)=M_{n} L f(x)$.
(c) $\lim _{n \rightarrow \infty} \lim _{k \rightarrow \infty} M_{n}\left(\psi_{k} * f\right)(x)=L f(x)$ a.e . 
The equalities in (a) are by (2.9) of [3] (or our Remark (2.2), or (2.5. iii)), (b) is a result of

$$
\left|M_{n} L f(x)-M_{n}\left(\psi_{k} * f\right)(x)\right| \leqq \frac{1}{\lambda\left(\mathfrak{B}^{n}\right)} \| \zeta\left(\pi^{-n}(y-x)\left\|_{r^{\prime}}\right\| L f-\psi_{k} * f \|_{r}\right.
$$

and (c) combines (a) and (b). (Interchange of limits in (c) gives the statement of pointwise convergence.) Using Fubini's theorem and appropriate translations, (b) can be written

$$
\begin{aligned}
M_{n} L f(x) & =\lim _{k \rightarrow \infty} \frac{1}{\lambda\left(\mathfrak{P}^{n}\right)} \int_{K^{d}} f(w) \int_{K^{d}} \zeta\left(\pi^{-n}(x-y)\right) \psi_{k}(y-\omega) d y d w \\
& =\lim _{k \rightarrow \infty} \frac{1}{\lambda\left(\mathfrak{P}^{n}\right)} \int_{K^{d}} f(w) \int_{K^{d}} \zeta(y) \psi_{k}\left(\pi^{-n}(x-w)-y\right) d y d w .
\end{aligned}
$$

The inner integral in the last expression is $\psi_{k} * \zeta\left(\pi^{-n}(x-w)\right)$. Since $\zeta \in S$ with $m=0, L \zeta=\psi_{k} * \zeta=\psi_{0} * \zeta$ for all $k \geqq 0$. Thus

$$
\begin{aligned}
& M_{n} L f(x)-\psi_{n} * f(x) \\
& \quad=\frac{1}{\lambda\left(\mathfrak{P}^{n}\right)} \int_{K^{d}} f(w)\left[L \zeta\left(\pi^{-n}(x-w)\right)-\psi_{0}\left(\pi^{-n}(x-w)\right] d w\right. \\
& \quad=M_{n}(\eta, f)(x),
\end{aligned}
$$

where $\eta(x)=L \zeta(x)-\psi_{0}(x)$. Supposing that (2.5.iii) holds and letting $n$ go to $\infty$ in (11), we have

$$
\lim _{n \rightarrow \infty} \psi_{n} * f(x)=f(x) \int_{K^{d}} \eta d \lambda+L f(x) \quad \text { a.e. , }
$$

proving that $\int \eta d \lambda=0$ and that $\lim _{n \rightarrow \infty} \psi_{n} * f(x)=L f(x)$ a.e. A condition for the validity of (2.5.iii) is simply " $\varphi \in \mathbb{R}_{1}$ ". Since

$$
\eta(x)= \begin{cases}\frac{1}{|x|^{d}} \int_{\mathfrak{F}^{0}}[\omega(x-y)-\omega(x)] d y \text { if } x \in\left(\mathfrak{P}^{0}\right)^{\prime} & (|x|>1) \\ \int_{|y-x|=1} \omega(x-y) d y-\omega(x) \quad \text { if }|x|=1 & \\ 0 \quad \text { if } x \in P^{1}, & (|x|<1)\end{cases}
$$

the condition " $\varphi \in \mathbb{R}_{1}$ " is seen to be implied by our hypothesis (iii).

Using (11) and (2.5.i), we have

$$
\left|\psi_{n} * f(x)\right| \leqq\|\varphi\|_{1} M(|f|)+M_{n}(|L f|)(x) .
$$

The bound (iv) follows from (2.4). That the operator $L^{*}$ is weak type (1.1) goes much as in part II of the proof. For $f \in \mathbb{R}_{1}$, we split $f$ into $h+g$ as before and obtain the inequality 


$$
\lambda(L * h(x)>t) \leqq \frac{a}{t^{2}} \int_{K^{d}}[f]_{t}^{2} d \lambda+b \lambda\left(D_{t}\right)
$$

from the fact that $L^{*}$ is strong type $(2,2)$. The supremum of the absolute value of the left side of (8) over $k>0$ is dominated by

$$
\sum_{P^{\prime} t} \int_{x_{m n}+\mathfrak{F}^{n}}|g(y)|\left[\frac{\left|\omega(x-y)-\omega\left(x-x_{m n}\right)\right|}{\left|x-x_{m n}\right|^{d}}\right] d y .
$$

The argument following (8) is then unaltered if $\psi_{k} * g$ is replaced by $L * g$, and results in

$$
\lambda\left(L^{*} g(x)>t\right)<c \lambda\left(D_{t}\right) .
$$

By the sublinearity of $L^{*}$, we have

$$
\left\{L^{*} f(x)>t\right\} \subset\left\{L^{*} g(x)>\frac{1}{2} t\right\} \cup\left\{L^{*} h(x)>\frac{1}{2}\right\} .
$$

Hence, $L^{*}$ is weak type $(1,1)$.

It remains to prove pointwise convergence for $\mathfrak{Q}_{1}$-functions. For $f \in \mathbb{R}_{1}^{+}$and $t>0$, decompose $f$ as when obtaining measure estimates: $f=g+h$. Thus, $\psi_{k} * f=\psi_{k} * h+\psi_{k} * g$. We know that $\lim _{k \leftarrow \infty} \psi_{k} * h$ exists a.e., because $h \in \mathbb{R}_{2}$. We have proved that $\lim _{k \rightarrow \infty} \psi_{k} * g$ exists in $D_{t}^{\prime}$; hence, for every $t>0 \lim _{k \rightarrow \infty} \psi_{k} * f(x)$ exists a.e. in $D_{t}^{\prime}$. Let $\varepsilon>0$. By (5.b) there is a $t$ such that $\lambda\left(D_{t}\right)<\varepsilon$. Hence,

$$
\lambda\left(\left\{x \in K^{d}: \lim _{k \rightarrow \infty} \psi_{k} * f(x) \text { does not exist }\right\}\right)<\varepsilon .
$$

Thus $\lim _{k \rightarrow \infty} \psi_{k} * f$ exists a.e., if $f \in \mathbb{Z}_{1}^{+}$. Clearly the pointwise limit will also exist for arbitrary $f \in \mathfrak{R}_{1}$, and the proof is complete.

A variant of the the theorem follows.

THEOREM 3.2. Under the hypothesis of (3.1) with (3.1.iii) replaced with

$$
\sum_{j=1}^{\infty}\left[\int_{\mathfrak{F}^{0} 0} \int_{\Sigma_{0}^{0}}\left|\omega\left(x+\pi^{j} y\right)-\omega(x)\right|^{2} d x d y\right]^{1 / 2}<\infty,
$$

the operators $\psi_{k} * f$ converge pointwise a.e. for $f \in \mathbb{Z}_{r}, 1 \leqq r<\infty$.

Proof. Parts I, II, and III of the proof are unchanged. In IV, we use (3.2.iii) to verify (2.5.ii) for the function $\eta=L \zeta-\psi_{0}$ and $r=$ 2. Condition (2.5.ii) is equivalent to

$$
\sum_{j=1}^{\infty}\left[\left.\int_{\mathfrak{D}^{-j}} \int_{\mathfrak{P}_{\mathfrak{S}}^{0}} \frac{[\omega(x-y)-\omega(x)]}{|x|^{d}} d y\right|^{2} d x\right]^{1 / 2} \lambda\left(\mathfrak{F}^{-j}\right)^{1 / 2}<\infty,
$$

and the terms of this series are dominated by those of (3.2.iii). The 
argument of IV thus proves pointwise convergence a.e. of $\psi_{k} * f$ for $f \in \mathfrak{R}_{2}$. If $1 \leqq r \leqq 2$, pointwise convergence follows for $f \in \mathfrak{R}_{r}$, as in the last paragraph of IV (that argument is valid for $f \in \mathbb{R}_{r}, 1 \leqq r \leqq 2$ ).

If $r>2$, then condition (3.2.iii) implies the same condition with 2 replaced by $r^{\prime}$; and, the condition with $r^{\prime}$ implies (2.5.ii). Thus, (2.5.iii) is again valid, and pointwise convergence follows.

The condition (3.2.iii) together with (2.5.iv) and (2.4) yield a certain $L_{2}$-estimate for the maximal singular integrals. If we assume a little more, we get the estimate for all $L_{r}$ funcnions, as follows.

THEOREM 3.3. Under the assumptions of (3.1) with (3.1.iii) replaced by

$$
\sum_{j=1}^{\infty}\left[\int_{\mathfrak{P}^{0}} \int_{\mathfrak{\Omega}^{0}}\left|\omega\left(x+\pi^{j} y\right)-\omega(x)\right|^{r^{\prime}} d x d y\right]^{1 / r^{\prime}}<\infty
$$

we have

$$
\begin{aligned}
{\left[\int_{E}\left|L^{*} f\right|^{r} d \lambda\right]^{1 / r} \leqq } & {\left[\frac{k_{1}}{k} \lambda(E)+\frac{k_{2}}{(1-k)} \int_{K^{d}}|f|^{r} \log ^{+}|f|^{r} d \lambda\right]^{1 / r} } \\
& +k_{3}\|f\|_{r} .
\end{aligned}
$$

For each $r \in] 1, \infty\left[\right.$, the $k_{i}$ 's are constants and the inequality is valid for $f \in \mathbb{R}^{r}, E$ measurable, and $\left.k \in\right] 0,1[$.

Proof. The condition (3.3.iii) gives (2.5.ii) for $r^{\prime}$ and $\eta=L \zeta-\psi_{0}$, as above. Hence (2.5.iii) and (2.5.iv) hold, so that $\lim _{n \rightarrow \infty} \psi_{n} * f$ exists pointwise a.e. and

$$
\left|\psi_{n} * f(x)-M_{n} L f(x)\right| \leqq b\left[M\left(|f|^{r}\right)(x)\right]^{1 / r}, \quad b \text { constant } .
$$

This gives

$$
\left|\psi_{n} * f\right| \leqq b\left[M\left(|f|^{r}\right)\right]^{1 / r}+M_{n}(|L f|), \quad \text { all } n ;
$$

and so $\left[\left|L^{*} f\right|-M(|L f|)\right]^{r} \leqq b M\left(|f|^{r}\right)$. Using a subscript $E$ to denote norms taken over the set $E$, we have

$$
\begin{aligned}
\left\|\left|L^{*} f\right|-M(|L f|)\right\|_{r, E} & \leqq b\left[\int_{E} M\left(|f|^{r}\right) d \lambda\right]^{1 / r} ; \\
\left\|L^{*} f\right\|_{r, E} & \leqq b\left[\int_{E} M\left(|f|^{r}\right) d \lambda\right]^{1 / r}+\|M(|L f|)\|_{r} .
\end{aligned}
$$

Application of (2.4.ii) to the $\Omega_{1}$ function $|f|^{r}$ together with (2.4.i) completes the proof.

REMARKS 3.4. (a) Condition (3.1.iii) can be written 


$$
\int_{\left(\mathfrak{P}^{0}\right)^{\prime}} \frac{\gamma\left(\pi^{-s(x)}\right)}{|x|^{d}} d x<\infty ; \gamma(x) \equiv \sup _{|z|=1} \int_{\mathfrak{P}^{0} 0}\left|\omega\left(z+\pi^{-s(x)} y\right)-\omega(z)\right| d y .
$$

The function $\gamma$ is somewhat analogous to the $\gamma$ used in [6] to prove $\mathfrak{R}_{r}$-convergence. Here we use the $L_{1}$-norm for $\left[\omega\left(x+\pi^{j} y\right)-\omega(x)\right]$ on $\mathfrak{P}^{0}$, so the condition $|\omega(x+y)-\omega(x)| \leqq \lambda(y)$ [used in [6]] does not necessarily hold. Use of the $\mathfrak{R}_{1}$-norm clearly gives us a weaker hypothesis. Both (3.1.i) and (3.1.iii) are implied by the condition

$$
\sum_{j=1}^{\infty} \sup \left\{\left|\omega\left(x+\pi^{j} y\right)-\omega(x)\right|: y \in \mathfrak{P}^{0}, x \in \mathfrak{D}^{0}\right\}<\infty,
$$

which is the Dini condition of [2], $\int_{0}^{1}\{\omega(t)\} /(t) d t<\infty$, in a convenient local field form.

(b) It would be interesting to weaken the hypothesis to one condition:

$$
\sum_{j=1}^{\infty} \int_{\mathfrak{F}^{0}} \int_{\mathfrak{Q}^{0}}\left|\omega\left(x+\pi^{j} y\right)-\omega(x)\right| d y d x<\infty .
$$

This condition implied by each of (3.1.i), (3.1.iii), (3.2.iii), (3.3.iii), and (3.4.i).

(c) The hypothesis in (3.13) of [6] is equivalent to the following statement: There is an $m \in Z^{+}$such that $\omega\left(x+\mathfrak{P}^{m}\right)=\{\omega(x)\}$ for all $x \in \mathfrak{Q}^{\circ}$. This condition and homogeneity imply that $\omega\left(x+\mathfrak{P}^{m+n}\right)=\{\omega(x)\}$ if $x \in \mathfrak{Q}^{n}$. In particular (i) above is trivial, and the $\mathfrak{Q}_{r}$-results of [6] are included in (3.1). For such an $\omega$ the proof of pointwise convergence simplifies, as follows. Again write $M_{n} L f-\psi_{n} * f=M_{n}(\eta, f)$, with $\eta$ given by (12) of the proof. If $x \in \mathfrak{\Omega}^{w}$ with $w \leqq-m$, then $\omega\left(x+\mathfrak{B}^{0}\right)=$ $\{\omega(x)\}$. Thus, (12) shows that $\eta=0$ on $\mathfrak{P}^{1} \cup\left(\mathfrak{P}^{-m+1}\right)^{\prime}$. We have

$$
\begin{aligned}
& \left|M_{n}(\eta, f)(x)-f(x) \int \eta d \lambda\right| \\
& \quad \leqq \frac{1}{\lambda\left(\mathfrak{P}^{n}\right)} \int_{\left(\mathfrak{B}^{n+1}\right)^{\prime} \cap \mathfrak{F}^{-m+n+1}}\left|\eta\left(\pi^{-n} y\right)\right||f(x+y)-f(x)| \mathrm{dy} .
\end{aligned}
$$

Since $\eta$ is bounded, the limit as $n$ goes to $\infty$ of the right side is zero for $x$ in the Lebesgue set. This proves that $\lim _{n \rightarrow \infty} \psi_{n} * f(x)$ exists a.e.

3.5. Singular integrals with $\mathfrak{\Omega}_{1}$-kernel. In (3.1), the condition $\omega \in \mathfrak{R}_{\infty}\left(K^{d}\right)$ can actually be replaced with the weaker condition $\omega \in \mathbb{R}_{1}\left(Q^{0}, \lambda\right)$, but at the expense of using Fourier transforms and convolutions of distributions, and other complications. In this subsection we will outline this method, which is due to Hörmander [5] in the $R^{n}$ case.

The fundamental space of test functions for distributions on $K^{d}$ 
is $\subseteq$; its dual is denoted by $\mathfrak{S}^{\prime}$. The assumption $\omega \in \mathfrak{R}_{1}\left(Q^{0}, \lambda\right)$ implies that $\psi_{k} \in \mathfrak{R}_{1, \mathrm{loc}}, k \geqq 0$, but we do not know, from general principles, that $\psi_{k} * f$ is well defined for $f \in \mathfrak{Q}_{r}$. The cancellation property $\int_{\mathfrak{D}_{0}^{0}} \omega d \lambda=0$ can be used to prove the existence of $\psi_{k} * f$. Let $\psi=\psi_{0}$. Then, $\psi$ defines an element $\langle\psi\rangle$ of $\sigma^{\prime}$ by $\langle\psi\rangle(\varphi)=\int_{K^{d}} \psi \varphi d \lambda$ and $\langle\psi\rangle$ has a Fourier transform defined by $\langle\psi\rangle^{\wedge}(\varphi)=\int_{K^{d}} \psi \hat{\varphi} d \lambda$. Let $\tau_{v}$ denote translation by $v: \tau_{v} f(x)=f(x-v)$. Some elementary computation establishes the equality

$$
\left\langle\tau_{v} \psi-\psi\right\rangle^{\wedge}(\varphi)=\langle\psi\rangle^{\wedge}\left(\varphi s_{v}\right), \varphi \in S,
$$

where $s_{v}(x)=\overline{\chi(x, v)}-1$. The hypothesis (3.2.i) implies that

$$
\left(\tau_{v} \psi-\psi\right) \in \mathfrak{R}_{1}\left(K^{d}\right) \text { for } v \in \mathfrak{P}^{0}
$$

since $\omega \in \mathbb{R}_{1}\left(Q^{0}, \lambda\right),\left(\tau_{v} \psi-\psi\right)$ is therefore in $\mathbb{R}_{1}\left(K^{d}\right)$ for all $v$. Thus

$$
\left(\tau_{v} \psi-\psi\right)^{\wedge} \in \widetilde{C}_{0}\left(K^{d}\right) \text { and }\left\langle\left(\tau_{v} \psi-\psi\right)^{\wedge}\right\rangle=\left\langle\tau_{v} \psi-\psi\right\rangle^{\wedge}
$$

for each $v$. Furthermore, $\left(\tau_{v} \psi-\psi\right)^{\wedge}(y)$ is uniformly bounded for $(v, y) \in \mathfrak{S}^{0} \times K^{d}$. There is a constant $M$ such that for any $x \neq 0$ there is a neighborhood $U(x)$, not containing 0 , such that $\left|s_{v}(y)\right| \geqq M$ for all $y \in U(x)$. Clearly the choice of $v$ for small $x$ must be large. But, if $x \in\left(\mathfrak{B}^{0}\right)^{\prime}$, then $v$ can be chosen in $\mathfrak{P}^{\circ}$. Define

$$
S_{x}=\frac{\left(\tau_{v} \psi-\psi\right)^{\wedge}}{s_{v}} \text { on } U(x)
$$

Then $S_{x}$ is a bounded continuous function on $U(x)$ and $\langle\psi\rangle^{\wedge}=\left\langle S_{x}\right\rangle$ on $U(x)$; (i.e., $\langle\psi\rangle^{\wedge}(\varphi)=\left\langle S_{x}\right\rangle(\varphi)$ if $\left.\operatorname{supp} \varphi \subset U(x)\right)$. The function $S(x)=S_{x}(x)(x \neq 0)$ satisfies $S(x)=S_{y}(x)$ if $x \in U(y)$. Thus $S$ is a continuous function on $K^{d} \backslash\{0\}$ such that for all $x \in K^{d} \backslash\{0\}$ there is a a neighborhood of $x$ such that $\langle\psi\rangle^{\wedge}=\langle S\rangle$ on the neighborhood. The function $S$ is bounded on $\left[\mathfrak{P}^{0}\right]^{\prime}$, for if $y \in\left(\mathfrak{P}^{0}\right)^{\prime}$ then the corresponding $v$ in (1) can be in $\mathfrak{P}^{\circ}$. The denominator is bounded away from zerouniformly in $v$. If $y \in \mathfrak{P}^{0} \backslash\{0\}$, say $y \in \mathfrak{\Omega}^{s-1}$, then an elementary argument shows that we must have $S\left(\pi^{-s} y\right)=S(y)$. Thus $S$ is bounded on $K^{d} \backslash\{0\}$ and defines a distribution $\langle S\rangle$ on $K^{d}$ such that $\langle\psi\rangle^{\wedge}=\langle S\rangle$ locally on $K^{d} \backslash\{0\}$. It follows that $\langle\psi\rangle^{\wedge}=\langle S\rangle$ everywhere on $K^{d} \backslash\{0\}$, and hence that $\operatorname{supp}\left[\langle\psi\rangle^{\wedge}-S\right] \subset\{0\}$. The cancellation property $\int_{0_{0}^{0}} \omega d \lambda=0$ can now be used to prove that $\langle\psi\rangle^{\wedge}-\langle S\rangle=0$; i.e., that $\langle\psi\rangle^{\wedge}=\langle S\rangle$ on $K^{d}$.

Arguments analogous to those given in the proof of (3.1) show that $\left\{\left\langle\psi_{k}\right\rangle^{\wedge}\right\}_{k=1}^{\infty}$ is actually a uniformly bounded sequence of functions which converge to a function $\Phi$, and that the differences $\left\langle\psi_{k}\right\rangle^{\wedge}-{ }_{n} \hat{\psi}_{k}$ 
are uniformly bounded. The inversion argument proves the $\Omega_{2}$-existence of $L_{k} f=\lim _{n \rightarrow-\infty} \psi_{k} * f$ and $L f=\lim _{k \rightarrow \infty} L_{k} f$ for $f \in \mathbb{S}_{2}$ and also the bound $\left\|L_{k} f\right\|_{2} \leqq A_{2}\|f\|_{2}$.

In obtaining weak type $(1,1)$ and other measure estimates, we don't know that $\psi_{k} * g$ (or even $L_{k} g$ ) exists for $g \in \mathbb{R}_{r}(1 \leqq r<2)$. However, we actually proved, using only (3.1.i) and $\omega \in \Omega_{1}\left(\mathfrak{Q}^{0}, \lambda\right)$, that the infinite series

$$
\sum_{P_{t}} \int_{x_{m n}+\mathfrak{F}^{n}}|g(y)| \int_{K^{d}} \xi_{\left(x_{m n}+\mathfrak{F}^{n}\right\rangle^{\prime}}(x) \frac{\left|\omega(x-y)-\omega\left(x-x_{m n}\right)\right|}{\left|x-x_{m n}\right|^{d}} d x d y
$$

is finite. (see II of proof of (3.1)). "Fubini" arguments combined with the analysis already given in II show that

$$
\int_{D^{t}} g(y)\left[\frac{\omega(x-y)-\omega\left(x-x_{m n}\right)}{\left|x-x_{m n}\right|^{d}}\right] d y
$$

exists absolutely for almost all $x \in D_{t}^{\prime}$. Thus

$$
\psi_{k} * g(x)=\int_{K^{d}} g(y) \psi_{k}(x-y) d y
$$

exists (the integral is proper) a.e. in $D_{t}^{\prime}$ for each $t>0$ and satisfies $\int_{D_{t}^{\prime}}\left|\psi_{k} * g(x)\right| d x \leqq M \int_{D_{t}}|g| d \lambda$.

It is apparently difficult to handle $g$ directly on $D_{t}$, but if we let $f \in \mathfrak{S}$, then $\psi_{k} * f$ and $\psi_{k} * g$ exist everyplace and, by the above analysis, the weak estimate

$$
\lambda\left(E_{t}\left[\psi_{k} * f\right]\right) \leqq \frac{c}{t}\|f\|_{1}
$$

is satisfied $(f \in \mathfrak{S})$. For arbitrary $f \in \mathbb{R}_{1}$, let $\lim _{n \rightarrow \infty}\left\|f-f_{n}\right\|_{1}=0, f_{n} \in \mathfrak{S}$. The estimate (2) implies that $\left(\psi_{k} * f_{n}\right)$ converges in measure; call its limit $L_{k} f$. Then $L_{k} f$ also satisfies (2), and we get the operators $L_{k}$ uniformly weak type $(1,1)$.

For $1<r<2$, the Marcinkiewicz interpolation theorem applies as before to give the estimates $\left\|L_{k} f\right\|_{r} \leqq A_{r}\|f\|_{r}$. The $L_{r}$-convergence argument is the same, for " $\psi_{k} \in \Omega_{1}$, loc" is all that was required. The duality proof is the same. Pointwise convergence is also valid; we needed only $\omega \in \mathfrak{R}_{1}\left(Q^{0}, \lambda\right)$, (3.1.iii), and $\mathfrak{R}_{r}$-convergence.

3.6. Two examples. (i) If $d=1, \mathfrak{\Omega}^{0}$ is a multiplicative group whose Haar measure is $\lambda$. For a nontrivial character $\omega$ of $\mathfrak{\Omega}^{0}, \int_{\mathfrak{Q}^{0}} \omega d \lambda=$ 0 holds. Extend $\omega$ to $K \backslash\{0\}$ by $\omega(x)=\omega\left(x^{*}\right)$. Since $Q^{0}$ is zerodimensional, $\omega$ vanishes on some neighborhood of 1 (see [4] or [9]). It follows that $\omega\left(x+\mathfrak{P}^{m}\right)=\{\omega(x)\}$ for all $x \in \mathfrak{\Omega}^{0}$, for some $m$. Hence, 
all hypothesis' of (3.1) hold for $\omega$ (see also (3.4.c)). The analogue of $\mathfrak{D}^{0}$ for the complex numbers is the circle group $T$; a character $e^{i n t}$ of $T$ defines a two-dimensional Calderón-Zygmund singular integral. In the case of the real numbers, the analogue of $Q^{0}$ is the multiplicative group $\{-1,1\}$; the real analogue of the singular integral is the Hilbert transform.

(ii) Let $P$ be a subset of $K^{d}$ satisfying

$$
P \cap-P=\varnothing \text { and } P \cup-P=K \backslash\{0\} \text {. }
$$

If $\omega$ satisfies $\omega(-x)=-\omega(x)$, then $\int_{\mathfrak{Q}^{0}} \omega(x) d x=0$. The convolutions are then given by

$$
\psi_{k} * f(y)=-\int_{P \cap\left(\Re^{k+1}\right)} \frac{\omega(x)}{|x|^{d}}[f(y+x)-f(y-x)] d x .
$$

In particular, we could set $\omega(x)=1$ if $x \in P$ and $\omega(x)=-1$ if $x \in-P$, and get another (different) analogue of the classical Hilbert transform. The conditions (3.1.i) and (3.1.iii) become conditions on $P$. For a further discussions of kernels of this type in the $p$-adic and $p$-series cases, see [6], (4.1) and (4.2).

4. Appendix by Keith Phillips. Maximal functions for a class of noncompact groups. In this section we give a general treatment of maximal functions. The entire section is independent of the rest of the paper. Our standing hypothesis is that $G$ is a locally compact group (written multiplicatively) with left Haar measure $\lambda$ and $\left\{U_{n}: n \in Z\right\}$ is a family of relatively compact Borel sets in $G$ satisfying

(i) $U_{n+1} \varsubsetneqq U_{n}$ for all $n \in Z$ and $\lim \lambda\left(U_{n}\right)=\infty$;

(ii) $\left\{U_{n}: n \in Z^{+}\right\}$is a neighborhood base at $e$;

(iii) $\lambda\left(U_{n} U_{n}^{-1}\right)<C \lambda\left(U_{n}\right), C$ constant, $n \in Z$.

(iv) For each $n \in Z$ there is an integer $l(n)$ such that

$$
U_{l(n)} \supset U_{n}^{-1} U_{n} \text { and } U_{j} \not \supset U_{n}^{-1} U_{n} \text { if } j>l(n) \text {. }
$$

And, there is a constant $\alpha$ such that $\lambda\left(U_{l(n)}\right)<\alpha \lambda\left(U_{n}\right)$ for all $n \in Z$.

Conditions (i)-(iii) are those for a Borel $D^{\prime \prime}$-sequence in [3], except that we use a "doubly infinite" sequence. If the $U_{n}$ 's are symmetric or $G$ is Abelian condition (iv) implies (iii), with $C=\alpha$. We call a sequence satisfying (4.1) an $M$-sequence.

The following theorem is similar to (2.2) of [3]; the main difference is that the sets $U_{n}$ need not have bounded measure. The proof uses only (4.1.i)-(4.1.iii).

Covering Theorem 4.2. Let $\mathscr{Q}=\left\{x U_{n}: x \in G, n \in Z\right\}$. Suppose $E \subset G$ and $\mathscr{C}^{\dagger} \subset \mathscr{Q}$ satisfy 
(i) $\lambda\left(E U_{n}\right)<\infty$ for all $n \in Z$;

(ii) for each $x \in E$, there is an $n$ such that $x U_{n} \in \mathscr{U}^{\dagger}$;

(iii) $\left\{n: x U_{n} \in \mathscr{U}^{\dagger}\right.$ for some $\left.x \in E\right\}$ is bounded below. Then, there are sequences $\left(x_{k}\right)_{k=1}^{\prime \prime}(1 \leqq \mu \leqq \infty)$ in $E$ and $\left(n_{k}\right)_{k=1}^{\mu}$ in $Z$ such that

(iv) $\left\{x_{k} U_{n_{k}}\right\}_{k=1}^{\mu}$ is a pairwise disjoint family in $\mathscr{U}^{\dagger}$;

(v) $\lambda(E) \leqq C \sum_{k=1}^{\mu} \lambda\left(U_{n_{k}}\right)$.

Proof. Let

$$
n_{1}=\operatorname{Min}\left\{n: x U_{n} \in \mathscr{U}^{\dagger} \text { for some } x \in E\right\},
$$

and suppose $x_{1} U_{n_{1}} \in \mathscr{U}^{\uparrow}, x_{1} \in E$. Inductively, suppose $\left(x_{k}\right)_{k=1}^{p}$ in $E$ and $\left(n_{k}\right)_{k=1}^{p}$ in $Z$ satisfy

(1) $\left\{x_{k} U_{n_{k}}\right\}_{k=1}^{p}$ is pairwise disjoint in $\mathscr{Q}^{\dagger}$;

(2) $n_{k}=\operatorname{Min}\left\{n: x U_{n} \in \mathscr{U}^{\dagger}\right.$ and $x U_{n} \subset\left[\bigcup_{i=1}^{k-1} x_{i} U_{n_{i}}\right]^{\prime}$ for some $\left.x \in E\right\}$, $1 \leqq k \leqq p$.

If $p=1$, (1) and (2) are satisfied. If $E \subset \bigcup_{k=1}^{p} x_{k} U_{n_{k}} U_{n_{k}}^{-1}$, we take $\mu=$ $p$ and see that the lemma is proved. Otherwise, take

$$
x \in E \cap\left[\bigcup_{k=1}^{p} x_{k} U_{n_{k}} U_{n_{k}}^{-1}\right]^{\prime}
$$

and let $j$ be the smallest integer such that $x U_{j} \in \mathscr{C}^{\dagger}$. We will show that $x U_{j} \subset\left[\cup_{k=1}^{p} x_{k} U_{n_{k}}\right]^{\prime}$. First, if $\left(x U_{j}\right) \cap\left(x_{1} U_{n_{1}}\right) \neq \varnothing$, then

$$
x \in x_{1} U_{n_{1}} U_{j}^{-1} \subset x_{1} U_{n_{1}} U_{n_{1}}^{-1} ; \text { but , } x \in x_{1} U_{n_{1}} U_{n_{1}}^{-1}
$$

is a contradiction. If there are $k$ 's such that $\left(x U_{j}\right) \cap\left(x_{k} U_{n}\right) \neq \varnothing$, let $q$ be the least among them $(1<q \leqq p)$. Then the inclusion $x U_{j} \subset$ $\left[\bigcup_{i=1}^{q-1} x_{i} U_{n_{i}}\right]^{\prime}$ holds. By (2), the inequality $j \geqq q$ holds, and this inequality implies that $x \in x_{q} U_{n_{q}} U_{n_{q}}^{-1}$, a contradiction. We have thus proved that the set

$$
\left\{j: x U_{j} \in \mathscr{U}^{\dagger} \text { and } x U_{j} \subset\left[\bigcup_{k=1}^{p} x_{k} U_{n_{k}}\right]^{\prime} \text { for some } x \in E\right\}
$$

is nonvoid; and, we let $n_{p+1}$ be its minimum and $x_{p+1} U_{n_{p+1}}$ an element of $\mathscr{U}^{\dagger}$. Our induction step is thus completed.

We will prove that $E \subset \bigcup_{k=1}^{h} x_{k} U_{n_{k}} U_{n_{k}}^{-1}$. This inclusion, which is already established if $\mu<\infty$, will clearly imply (v). First, we must have $\lim _{k \rightarrow \infty} n_{k}=\infty$, for

$$
\sum_{k=1}^{\infty} \lambda\left(U_{n_{k}}\right)=\sum_{k=1}^{\infty} \lambda\left(x_{k} U_{n_{k}}\right)<\lambda\left(E U_{n_{1}}\right)<\infty .
$$

Hence, for given $x \in E$ and $x U_{p} \in \mathscr{U}^{\dagger}$ we must have $\left(x U_{p}\right) \cap\left(x U_{n_{k}}\right) \neq \varnothing$ for some $k$ such that $n_{k} \leqq p$; if not, $x$ and $p$ would have been selected 
instead of $x_{j}$ and $n_{j}$ as soon as $n_{j}$ exceeded $p$. If $q$ is any integer such that $\left(x U_{p}\right) \cap\left(x_{q} U_{n_{q}}\right) \neq \varnothing$ and $n_{q} \leqq p$, then we obtain $x \in x_{q} U_{n_{q}} U_{n_{q}}^{-1}$.

Let $\mathscr{C}^{+}$denote the positive (finite) regular Borel measures on $G$. If $f$ is locally integrable and $\mu \in \mathscr{C}^{+}$, let

$$
\begin{array}{ll}
M_{n} f(x)=\frac{1}{\lambda\left(U_{n}\right)} \int_{x T_{n}} f d \lambda, n \in Z ; & M f(x)=\sup \left\{M_{n} f(x): n \in Z\right\} \\
M_{n} \mu(x)=\frac{\mu\left(x U_{n}\right)}{\mu\left(U_{n}\right)}, n \in Z ; & M \mu(x)=\sup \left\{M_{n} \mu(x): n \in Z\right\} .
\end{array}
$$

For a nonnegative function $g$ and $t>0$, let $E_{t}[g]=\{x: g(x)>t\}$.

4.4. First weak type estimate. For an $M$-sequence $\left\{U_{n}\right\}_{n \in Z}$,

(i ) $\lambda\left(E_{t}[M f]\right) \leqq \frac{C}{t} \int_{G} \xi_{E_{t / \alpha}[M f]} f d \lambda$;

(ii) $\lambda\left(E_{t}[M \mu]\right) \leqq \frac{C}{t} \mu\left(E_{t / \alpha}[M \mu]\right)$

hold for all $f \in \mathfrak{R}_{r}(1 \leqq r<\infty), \mu \in \mathscr{K}^{+}$, and $t>0$.

Proof. We concentrate on the proof for $M f ; M \mu$ is similar. Let $t$ be fixed and define

$$
\mathscr{U}^{\dagger}=\left\{x U_{n}: t \lambda\left(U_{n}\right)<\int_{x U_{n}} f d \lambda\right\} .
$$

Let $E$ be any compact subset of $E_{t}[M f]$. It is clear that (4.2.i) and (4.2.ii) are satisfied for the pair $\left(\mathscr{C}^{\dagger}, E\right)$. Condition (4.2.iii) is also satisfied, for there cannot be sequences $\left(x_{i}\right)_{i=1}^{\infty}$ and $\left(n_{i}\right)_{i=1}^{\infty}$ such that $\lim _{i \rightarrow \infty} n_{i}=-\infty$ and $\int \xi_{x_{i} u_{n_{i}}} f d \lambda>t \lambda\left(U_{n_{i}}\right)$. This assertion is obvious if $r=1$ (and also for $\mu$ ) and is a consequence of Hölder's inequality if $r>1$. We thus obtain

$$
\lambda(E) \leqq C \sum_{k=1}^{\infty} \lambda\left(U_{n_{k}}\right)<\frac{C}{t} \int_{V} f d \lambda,
$$

where $\left(x_{k} U_{n_{k}}\right)_{k=1}^{\infty}$ is the sequence guaranteed by (4.2) and $V=\bigcup_{k=1}^{\infty} x_{k} U_{n_{k}}$. If $x \in V$, then $x_{k}^{-1} x \in U_{n_{k}}$ for some $k$, so that $x_{k}^{-1} x U_{l\left(n_{k}\right)} \supset U_{n_{k}}$. Thus:

$$
\begin{aligned}
\frac{1}{\lambda\left(U_{l\left(n_{k}\right)}\right)} \int \xi_{x U^{l} l\left(n_{k}\right)} f d \lambda & \geqq \frac{\alpha^{-1}}{\lambda\left(U_{n_{k}}\right)} \int \xi_{x U^{t} l\left(n_{k}\right)} f d \lambda \\
& \geqq \frac{\alpha^{-1}}{\lambda\left(U_{n_{k}}\right)} \int \xi_{x_{k} U^{\prime} n_{k}} f d \lambda>\alpha^{-1} t .
\end{aligned}
$$

We have proved that $V \subset E_{t / \alpha}[M f]$; hence, by (1),

$$
\lambda(E) \leqq \frac{C}{t} \int \xi_{E_{t / \alpha}[M f]} f d \lambda .
$$


The inequality (i) follows, for the right side in (2) does not depend on $E$ and the supremum of the left side over compact $E$ contained in $E_{t}[M f]$ is $\lambda\left(E_{t}[M f]\right)$.

Before stating integral estimates, we first show how to get a different measure estimate, from (4.4).

4.5. Second weak type estimate. With the notation of (4.4),

$$
\lambda\left(E_{t}[M f]\right) \leqq \frac{C}{(1-k) t} \int \xi_{E_{k t}[f]} f d \lambda
$$

holds for every $k$ in] 0,1 .

Proof. Let $g=f \xi_{\{f>k t\}}$. Since

$$
M f \leqq M g+k t \quad \text { and } \quad E_{t}[M f] \subset E_{(1-k,}[M g],
$$

(4.4.i) gives us

$$
\lambda\left(E_{t}[M f]\right) \leqq \frac{C}{(1-k) t} \int_{E_{(1-k) t \alpha^{-1[M g]}}} g d \lambda=\frac{C}{(1-k) t} \int_{\left.E_{k t} \mid f\right\}} f d \lambda .
$$

(Notice how conveniently $\alpha$ disappeared.)

4.6. Integral Estimates. Notation is an (4.4).

(i ) $f \in \mathbb{R}_{r}^{+}(1<r<\infty) \Rightarrow\|M f\|_{r} \leqq \frac{r}{r-1} \min \left[(C r)^{1 / r}, C \alpha^{r-1}\right]\|f\|_{r}$.

(ii) $\left\{f \in \mathbb{R}_{1}^{+}, k \in\right] 0,1[, s \in] 0,1[, E \lambda$-measurable $\} \Rightarrow$

$$
\begin{aligned}
& \text { (a) } \int_{E}[M f] d \lambda \leqq \frac{\lambda(E)}{k}+\frac{C}{1-k} \int_{G} f\left[\log ^{+} f\right] d \lambda ; \\
& \text { (b) } \int_{E}[M f]^{s} d \lambda \leqq C^{s} \frac{\lambda(E)^{1-s}}{1-s}\left[\int_{G} f d \lambda\right]^{s} .
\end{aligned}
$$

Method of proof. In (i), the constant $(C r)^{1 / r}$ is obtained using estimates based on (4.5) and minimization with respect to $k ; C \alpha^{r-1}$ is obtained using estimates based on (4.4.i). See the proof of (3.2) in [7]. For (ii), (4.5) is used; see (3.4) of [7].

4.7. Ergodic groups. In [1], Calderón calls a locally compact group ergodic if there is a family $\left\{N_{r}\right\}$ of compact open symmetric neighborhoods of the identity, indexed by the real parameter $r>0$, such that $N_{r} N_{s} \subset N_{r+s}$ and $\lambda\left(N_{2 r}\right)<\alpha \lambda\left(N_{r}\right), \alpha$ constant. If we take $U_{n}=N_{2^{-n}}$ we clearly get an $M$-sequence from an ergodic family $\left\{N_{r}\right\}$, for which $\alpha=C$. Let

$$
M^{*} f(x)=\sup \left\{\frac{1}{\lambda\left(N_{r}\right)} \int \xi_{x_{r}} f d \lambda: r>0\right\}
$$


If $2^{-n}<r \leqq 2^{-n+1}$, then

$$
\frac{1}{\lambda\left(N_{r}\right)} \int \xi_{x N_{r}} f d \lambda \leqq \frac{\alpha}{\lambda\left(U_{n-1}\right)} \int \xi_{x U_{n-1}} f d \lambda .
$$

Thus, $M^{*} f \leqq \alpha M f$, and we get a theorem like (4.6) for $M^{*} f$, but with an additional constant on the right. Actually one can do a little better, and obtain the same constant for $M^{*}$ as for $M$. To see this, observe that the inequality $M^{*} f \leqq \alpha M f$ implies that $E_{t}\left[M^{*} f\right] \subset E_{t / \alpha}[M f]$ for each $t>0$. Hence, we have

$$
\begin{aligned}
\lambda\left(E_{t}\left[M^{*} f\right]\right) & \leqq \lambda\left(E_{t / \alpha}[M f]\right) \leqq \frac{\alpha}{t} \int \xi_{E_{t / \alpha^{2[M f]}}} f d \lambda \\
& \leqq \frac{\alpha}{t} \int_{E_{t / \alpha^{2\left[M^{*} f\right]}} f d \lambda .}
\end{aligned}
$$

The second inequality holds by (4.4.i) and third because $M^{*} f \geqq M f$. We thus have a constant $\beta>0\left(\beta=\alpha^{-2}\right)$ such that

$$
\lambda\left(E_{t}\left[M^{*} f\right]\right) \leqq \frac{\alpha}{t} \int \xi_{t \beta^{\left[M^{*} f\right]}} f d \lambda .
$$

This inequality yields (4.6) with no additional constant $\alpha$ on the right, as indicated in (4.6).

Maximal functions for $\left(K^{d},+\right)$ are of course of the ergodic type. The additional condition that the $U_{n}$ 's are groups makes the theorems simpler.

4.7. Metrics groups. If the topology of $G$ is given by a left invariant metric $\rho$, then $\left\{S_{r}(0): r>0\right\}$ forms an ergodic family provided that $\lambda\left(S_{2 r}(0)\right) \leqq \alpha \lambda\left(S_{r}(0)\right)$. Maximal functions defined over spheres thus give a version of (4.4) and (4.5). In [10], K. T. Smith defines maximal functions for a class of metric spaces admitting measures satisfying certain conditions, namely (a), (b), (c), and (d) on the top of p. 159 of [10]. Smith's conditions (a), (c), (d) are satisfied for any locally compact metric group and a left Haar measure. The condition (b) [there is a constant $K$ such that $\lambda(S(x, 4 r)) \leqq K \lambda(S x, r))]$ implies that $\left\{S_{r}(0)\right\}$ is an ergodic family. Hence, for a locally compact metric group $G$ with left invariant metric, the proofs of (4.4) and (4.6) are different proofs of Smith's Theorem 1,2, and 3.

Maximal functions for certain metric spaces are also studied in [8], where the main interest is in applications to harmonic and analytic fuctions on relatively compact sets in $C^{n}$. The sets over which Rauch takes averages have bounded measures. 


\section{REFERENCES}

1. A. P. Calderón, A general ergodic theorem, Ann. of Math. 58 (1953).

2. A. P. Calderón and A. Zygmund, On the existence of certain singular integrals, Acta Math. 88 (1952), 85-139.

3. R. E. Edwards and Edwin Hewitt, Pointwise limits for sequences of convolution operators, Acta Math. 113 (1965), 181.

4. Edwin Hewitt and Kenneth Ross, Abstract harmonic analysis I, Springer, Heidelberg, 1963.

5. Lars Hörmander, Estimates for translation invariant operators in $L_{p}$ spaces, Acta Math. 104 (1960).

6. Keith Phillips, Hilbert transforms for the p-adic and p-series fields, Pacific J. Math. 23 (1967) 329.

7. - The maximal theorems of Hardy and Littlewood, Amer. Math. Monthly, 74 (1967), 648.

8. Rauch, Harmonic and analytic functions of several variables and the maximal theorems of Hardy and Littlewood, Canad. J. Math. 8 (1956), 171-183.

9. Paul Sally and Mitchell Taibleson, Special functions on locally compact fields, Acta Math. 116 (1966), 279.

10. K. T. Smith, A generalization of an inequality of Hardy and Littlewood, Canad. J. Math. 8 (1956), 157-170.

11. Mitchell Taibleson, Multipliers on local fields (to appear)

12. - Harmonic analysis on n-dimensional vector spaces over local fields, Math. Ann. 176 (1968), 191-207.

13. A. Zygmund, Trigonometric series, Vol. I and II, Cambridge, 1959.

Received April 10, 1968. Work of the first author was supported by NSF, Grant GP-7691 and of the second author by U.S. Army Research Office (Durham) contract no. DA-31-124-ARO(D)-58.

California Institute of Technology and New Mexico State University WASHINGTON UNIVERSITY 



\section{PACIFIC JOURNAL OF MATHEMATICS}

\section{EDITORS}

H. ROYDEN

Stanford University

Stanford, California

Richard Pierce

University of Washington

Seattle, Washington 98105
J. DugundJI

Department of Mathematics

University of Southern California

Los Angeles, California 90007

BASIL GORDON

University of California

Los Angeles, California 90024

\section{ASSOCIATE EDITORS}

E. F. BECKENBACH

B. H. NeUmanN

F. WOLF

K. YOSHIDA

\section{SUPPORTING INSTITUTIONS}

UNIVERSITY OF BRITISH COLUMBIA

CALIFORNIA INSTITUTE OF TECHNOLOGY

UNIVERSITY OF CALIFORNIA

MONTANA STATE UNIVERSITY

UNIVERSITY OF NEVADA

NEW MEXICO STATE UNIVERSITY

OREGON STATE UNIVERSITY

UNIVERSITY OF OREGON

OSAKA UNIVERSITY

UNIVERSITY OF SOUTHERN CALIFORNIA
STANFORD UNIVERSITY

UNIVERSITY OF TOKYO

UNIVERSITY OF UTAH

WASHINGTON STATE UNIVERSITY

UNIVERSITY OF WASHINGTON

$* * \quad * \quad *$
AMERICAN MATHEMATICAL SOCIETY
CHEVRON RESEARCH CORPORATION
TRW SYSTEMS
NAVAL WEAPONS CENTER




\section{Pacific Journal of Mathematics}

Vol. 30, No. $1 \quad$ September, 1969

William Wells Adams, Simultaneous diophantine approximations and cubic irrationals ..................................... 1

Heinz Bauer and Herbert Stanley Bear, Jr., The part metric in convex

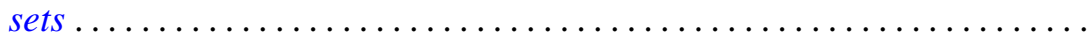

L. Carlitz, A note on exponential sums ...................... 35

Vasily Cateforis, On regular self-injective rings ................. 39

Franz Harpain and Maurice Sion, A representation theorem for measures on infinite dimensional spaces ......................... 47

Richard Earl Hodel, Sum theorems for topological spaces .............. 59

Carl Groos Jockusch, Jr. and Thomas Graham McLaughlin, Countable

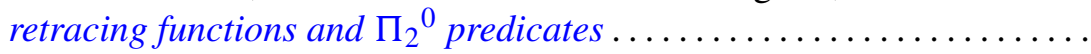

Bjarni Jónsson and George Stephen Monk, Representations of primary

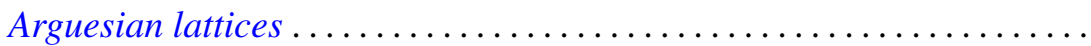

Virginia E. Walsh Knight, A continuous partial order for Peano continua...................................... 141

Kjeld Laursen, Ideal structure in generalized group algebras ........... 155

G. S. Monk, Desargues' law and the representation of primary lattices . . . 175

Hussain Sayid Nur, Singular perturbation of linear partial differential equation with constant coefficients ..........................

Richard Paul Osborne and J. L. Stern, Covering manifolds with cells ... 201

Keith Lowell Phillips and Mitchell Herbert Taibleson, Singular integrals in several variables over a local field...

James Reaves Smith, Local domains with topologically $T$-nilpotent radical....

Donald Platte Squier, Elliptic differential equations with discontinuous coefficients .................................

Tae-il Suh, Algebras formed by the Zorn vector matrix...

Earl J. Taft, Ideals in admissible algebras . .................... 259

Jun Tomiyama, On the tensor products of von Neumann algebras........ 263

David Bertram Wales, Uniqueness of the graph of a rank three group ..... 271

Charles Robert Warner and Robert James Whitley, A characterization of regular maximal ideals ......................... 